\title{
Formación académica del psicólogo en la atención del adulto mayor: retos
}

\section{Academic training of the psychologist for caregiving of elders: challenges}

\author{
Formação acadêmica do psicólogo \\ em cuidados deadulto idoso: desafios
}

\author{
Trino Javier Gascón González', \\ https://orcid.org/0000-0003-3575-8535
}

https://doi.org/10.53287/bupm9814vy38c

Fecha de ingreso: 16 de noviembre de 2020

Fecha de aprobación: 26 de marzo de 2021

Conflictos de interés: El autor declara no tener conflictos de intereses.

\section{RESÚMEN}

El propósito de la presente investigación fue realizar una revisión bibliográfica sobrela formación académica del psicólogo en la atención del adulto mayor, en Venezuela. Su pertinencia radica en, aportar información innovadora en esta área, por lo poco explorado del tema en el país. De allí que, es importante considerar los conocimientos y experiencias que posee el psicólogo, acerca de esta área del ciclo vital, desde un enfoque académico. El acceso a nuevos conocimientos permitirágenerar programasla atención psicológica especializada para los adultos mayores, tanto institucionalizados como no institucionalizados; procurando no solo mejorar la calidad de vida del adulto mayor; sino abarcarlo desde lasmuchas áreas de su funcionamiento integral. Concluyendo, podemos señalar que, existe la imperiosa necesidad de formaracadémicamente al psicólogoVenezolano en esta área dela Psicogerontología; a su vez descartando creencias que consideran al psicólogo como uno de los profesionales que posee todo el conocimiento científico, para atender a esta población.

\section{PALABRAS CLAVE}

Adulto Mayor; Formación Académica: Psicólogo; Educación; Psicogerontología.

1 Doctorado en Psicología, Universidad Católica Andrés Bello (UCAB), Caracas, Venezuela. Correo electrónico.: drtrinogascon@gmail.com 


\begin{abstract}
The purpose of this research paper was to carry out a bibliographical revision on the academic training of the psychologistfor the caregiving of elders, in Venezuela. Its relevance relays on providing innovating information in the area, for this subject has been little explored in this country. Therefore, it is important to consider all knowledge and experiences held by the professional psychologist on this stage of human development, from an academic perspective. The access to new knowledge will allow generating programs for appropriate psychological intervention directed to elders, whether these be institutionalized or non-institutionalized; aiming, not only to improve life quality of an old person, but also to address to it from the different areas of its overall functioning. To conclude, we can highlight that it actually exist the imperious need to train academically Venezuelan psychologist, as one of those professionals sufficiently competent with scientific knowledge to provide proper attention to this population.
\end{abstract}

\title{
KEY WORDS
}

Elders; AcademicTraining; Psychologist; Education; Psychogerontology.

\section{RESUMO}

O objetivo da presente investigação foi realizar uma revisão bibliográfica sobre a formação acadêmica do psicólogo no atendimento a idosos na Venezuela. Sua relevância está no fornecimento de informações inovadoras nessa área, devido ao pouco explorado da questão no país. Portanto, é importante considerar o conhecimento e as experiências que o psicólogo tem, sobre essa área do ciclo da vida, a partir de uma abordagem acadêmica. O acesso a novos conhecimentos permitirá que programas gerem atendimento psicológico especializado para idosos, institucionalizados e não institucionalizados; tentando não apenas melhorar a qualidade de vida dos idosos; mas cubra-o das muitas áreas de sua operação integral. Em conclusão, podemos destacar que há uma necessidade urgente de treinar academicamente o psicólogo venezuelano nessa área da psicogerontologia; por sua vez, descartando crenças que consideram o psicólogo um dos profissionais que possui todo o conhecimento científico, para atender a essa população.

\section{PALAVRAS-CHAVE}

Idoso; Formação Acadêmica; Psicólogo; Educação; Psicogerontologia.

\section{Introducción}

A medida que la sociedad crece aceleradamente, existe una tendencia mundial al envejecimiento de la población mundial, como lo estipulalo la resolución de la ONU (2020), donde proclamo la Década del Envejecimiento Saludable (2020-2030). Esta refiere que, la cifra de personas de 60 años o más habrá aumentado en un $34 \%$, de 1000 millones en 2019 a 1400 millones. Se espera que la población de adultos mayores, llegue en el 2050, será más del doble y alcanzará los 2100 millones. Por primera vez en la historia de la humanidad este grupo etario superará las cifras del grupo de la población infantil y adolescente, Actualmente hay más personas mayores que niños menores de 5 años. Entre el 2020 y el 2050, se espera que la población de personas de 60 años o más superaran en número a los adolescentes y jóvenes de edades comprendidas entre los 15 y los 24 años. 
Por lo tanto, el hecho de que la población de adulto mayor está aumentando, es una realidad y es importante garantizar la calidad de vida de estas personas. Así que, es pertinente fomentar la formación académica especializada; así como promover la investigación en esta área, que generen conocimientos, que aporten beneficios en la atención a la población adulo mayor. Como lo afirma Fernández-Ballesteros (2009b), la sociedad juega un papel trascendental con responsabilidad en la generación de condiciones y oportunidades de progreso en el desarrollo personal y profesional hacia el bienestar de las personas de la tercera edad. Por lo que se hace necesario resaltar, el déficit de profesionales especializados en el área de la Psicogerontología, Psicología del envejecimiento y adulto mayor.

Históricamente los estudios en el campo de la psicología de la vejez, se enmarcan en diferentes etapas históricas. Estas comienzan desde el siglo XVIII hasta el presente. Durante el siglo XVIII, la obra de Nicolás Tetens (1777, citada por L. Galvanoski), se encontró el primer trabajo serio en el campo del desarrollo adulto y envejecimiento. El mismo, pone de relieve la importancia de hallar leyes generales de desarrollo, que no se encuentran en otros períodos de edad. A partir de 1920, son más frecuentes los trabajos sobre el desarrollo adulto y el envejecimiento. Autores como Half (1922), son reconocidos como pioneros en la psicología de la vejez, en los Estados Unidos. Para 1922, publicó un libro en el que apunta la dependencia de las particularidades psicológicas de la edad o las causas biológicas; razón por la cual Munnichs (1966) lo llegó a catalogar como "el primer psicogerontólogo".

Half (1922) se oponía a la creencia muy difundida de que la vejez era el reverso del desarrollo de la adolescencia. Negándose a aceptar un "modelo deficitario", basado en el aumento de conocimientos y facul- tades en la juventud, el desarrollo máximo en la adultez media y la disminución de la capacidad junto con la involución, en la vejez. Procuró demostrar que la juventud y la vejez poseen su propio y específico modo de "sentir, pensar y querer". Además, se basa en los resultados de sus investigaciones para señalar que las diferencias individuales de la vejez son mucho mayores que las de la juventud. Este descubrimiento apoyado por los resultados de investigaciones puso de manifiesto la problemática del límite cronológico de las edades. Por otra parte, Ericsson (1902-1994), es considerado el autor más relevante, de tradición psicoanalítica que supone una reconceptualización de la dinámica evolutiva del ser humano. En su teoría del desarrollo psicosocial, identificó todas las etapas en función de las crisis del yo que describe para cada una de ellas; denominó la fase final con el término de vejez y señaló como crisis característica de esta etapa la relación entre la integridad del yo versus la desesperanza.

Para la década de los 90, surgen interesantes teorías y estudios que parten de un enfoque de la vejez como etapa vital; como una etapa más de la experiencia humana y que puede y debe ser considerada una fase positiva del desarrollo individual y social. De acuerdo con Moragas (2004), sociólogo dedicado al estudio de la gerontología social, considera que el estudio de la vejez debe hacerse desde una concepción amplia e integradora que supere los prejuicios sociales y actitudes negativas hacia la misma.

En este orden de ideas, Carbajo (2008) refiere que la psicología del envejecimiento es un campo poco investigado en Venezuela. En muchos departamentos de psicología, solamente imparten cursos sobre la etapa de la tercera edad o la gerontología a una población académica específica como médicos y enfermeras, dejando de la a otros profesionales que integran los 
equipos multidisciplinarios. De allí que, es de considerar que para enfrentar los problemas del envejecimiento, se hace necesaria la colaboración de equipos multidisciplinarios, conformados por médicos geriatras y gerontólogos, psicólogos, enfermeras, trabajadores sociales, entre otros. En función de lo planteado, este artículo de revisión se enfocó en la necesidad de formación académica del psicólogo Venezolano, en el área específica de la Psicogerontología. Lo que se constituye en una herramienta esencial en la atención del adulto mayor, con alta prevalencia de enfermedades mentales, muchas de ellos irreversibles (Conde, 1998). Siendo una de las alteraciones que experimenta el ser humano durante la última etapa del ciclo vitalcomo un cambio en sus funciones mentales, más significativas (De la Fuente, 1999).

La cuestión del envejecimiento se pudo ver en su complejidad cuando se introdujeron los aspectos concernientes a la subjetividad, es decir, el punto de vista del envejeciente y de su entorno más íntimo; cuando se comenzó a visualizar la implicancia subjetiva en el curso que adopta el propio envejecimiento y se desarrollaron distintas maneras de abordarlo, tanto a nivel preventivo como asistencial. Lo cual implicó el crecimiento del campo de la salud mental en el envejecimiento, conocido hoy en día como Psicogerontología.

Actualmente, está claro que no son sólo los avances médicos y tecnológicos los que mejoran la calidad de vida, sino la presencia de disciplinas sociales y humanas que colaboran en equipo para entender la complejidad del ser humano que envejece y lo ayudan a rehabilitarse. Asimismo, superar los prejuicios teóricos (Zarebski, 2005) permite ampliar este campo, del ámbito parcial y restringido de la clínica, a la prevención, la interdisciplina y la intersectorialidad. Lo cierto es que, empleando estrategias que contribuyan al cambio conductual, a la promoción y prevención de la enfermedad, se optimiza el proceso de adaptación con intervenciones que apuntan a necesidades propias de esta población; además de contexto familiar y social que lo rodea (Colegio Oficial de Psicólogos, 2002).

A lo anteriormente señalado, se suma la preocupación de los teóricos por implementar programas de intervención que no solo involucren al adulto mayor, sino también su contexto. Por lo tanto, el diseño de programas de formación en el área de la Psicogerontología, debe considerar no solo los factores físicos relacionados con el adulto mayor,tales como su historia personal, sino además factores psicosociales, que intervienen directamente en el proceso de adaptación y bienestar de los adultos mayores. (Fernández, 1992).

En este sentido, Moura, Navarro, Wolf \&Cabrol, (1998), han señalado que América Latina no se encuentra ajeno a esta inquietud de mejorar la educación profesional y los sistemas de certificación y acreditación académica. Particularmente en Chile, se viene trabajando con relación a la definición de competencias para carreras técnicas, desde hace más de dos décadas. Ahora bien, no obstante es a partir de los noventa, cuando la propuesta de la Formación Basada en Competencias es asumida por el Estado, a través del Ministerio de Educación, obedeciendoa la experiencia internacional de países desarrollados, lineamientos UNESCO y por directrices del Banco Mundial (Fundación Chile, 2000).

Como consecuencia, es así como en la actualidad se percibe que la acreditación de los programas educativos y de entrenamiento en Psicología, en la mayor parte de universidades de Norte América y Europa, buscan desarrollar al profesional con habilidades y competencias profesionales (Gutiérrez, 2005). Es de señalar que, la 
Psicología es una ciencia sustentada en la investigación y una profesión que estudia los procesos de desarrollo cognoscitivos, emocionales y sociales del ser humano.

A tal efecto, de acuerdo con el Libro Blanco de Psicología de España (2005), Las competencias especificas que se deben adquirir para el grado en psicología, siendo las más importantes: ser capaz de planificar y realizar una entrevista, ser capaz de describir y medir variables y procesos psicológicos, ser capaz de definir los objetivos y elaborar el plan de intervención, conocer y ajustarse a las obligaciones deontológicas de la psicología, "saber analizar necesidades y demandas de los destinatarios y "saber elegir las técnicas de intervención psicológica adecuadas, Siempre con el fin de promover el bienestar y la calidad de vida de las personas.

Claro está, el psicólogo puede intervenir profesionalmente en cualquiera de las etapas del ciclo vital de las personas; por ello debe tener conocimientos y habilidades necesarias para realizar el abordaje respectivo. Al mismo tiempo, los planes y programas de estudio deben tener en cuenta los cambios que se están generando producto del envejecimiento de la población. Es de considerar a la Psicogerontología como una especialidad de los psicólogos, con lo cual su desarrollo debe ir de la mano de la Psicogeriatría, especializando y legitimando así a los profesionales de la Psicología.

Por otra parte, Fernández-Ballesteros (2001), refiere que en la práctica gerontológica, la demanda del psicólogo es mínima y el intrusismo de otros profesionales en el trabajo psicológico es muy grande. Lo importante, en mi opinión, es tomar conciencia de este hecho y tratar de actuar en distintos frentes: 1) actualización de los planes de estudio universitarios para una sociedad que envejece, 2 ) investigación en el amplio abanico de temas y problema s relacionados con la vejez y el envejecimiento, 3) formación continuada y especializada del psicólogo, 4) demostración de la importancia del rol del psicólogo en los distintos contextos gerontológicos, y otras muchas actuaciones derivadas. A la hora de establecer una agenda de trabajo e iniciar un proyecto sobre las potenciales áreas de actuación del psicólogo, conviene presentar lo que a nivel internacional se está elaborando.

Lo cierto es que, se debe tener en cuenta que las intervenciones tenderán a ser más efectivas en la medida en que se aborde al adulto mayor desde su integralidad, trabajando junto con otras disciplinas y teniendo un amplio conocimiento académico y profesional sobre estas personas; un programa de intervención debe estar basado en indicadores biológicos, económicos, psicológicos y sociales ya que las inconsistencias se deben a miradas subjetivas del adulto mayor. (OMS, 2006; Uribe-Rodríguez, Molina \& González, 2006).

Lo antes expuesto nos lleva a proponer un programa como los que ofrece la Universidad de Salamanca, (2021/2022). Titulado: Máster Universitario en Psicogerontologia, el cual, posee una formación especializada en el campo de la Psicología del Envejecimiento con la finalidad de adquirir competencias profesionales e investigadoras para el desarrollo de tareas de evaluación, intervención psicológica y/o investigación con las personas mayores sanas, con deterioro cognitivo o con otras psicopatologías en distintos ámbitos familiares e institucionales.

Cabe destacar que, el psicólogo venezolano, como el de otras partes del mundo, tiene herramientas adquiridas en su formación para abordar y tratar a los adultos mayores, pero la excelencia que se busca alcanzar, está vinculada con las competencias específicas en el área, preparación académica y especialización para el abor- 
daje en el área del adulto mayor. Por lo tanto, que el psicólogo pueda o quiera participar en equipo multidisciplinario o individual en la atención de los adultos mayores es válido, pero es necesaria su formación académica especializada en el área del envejecimiento, al igual que cualquier otro profesional y en cualquier otra área.

Aunado a todo lo anterior, podemos adicionar que existe vacilación al saber que las políticas de estado Venezolano, están dirigidas a atender separadamente al adulto mayor, de manera Medico- SocialEconómica- Emocional; además que los variados programas sociales tanto públicos como privados, no logran la sustentabilidad. Sin embargo, el tiempo no se detiene y el proceso de envejecimiento continúa. La población anciana, unos más que otros, experimentará sentimiento frustración, insatisfacciones; además de tristeza y soledad (Reyes, 1996); por lo que se hace imperioso plantear desde todos los espacios científicos, la falta de profesionales académicamente acreditados, para la atención de las sociedades con adultos mayores relegados.

\section{Conclusiones}

Finalmente, el camino recorrido por los que nos antecedieron tiene queservirnos para mejorar. Mientras exista vida, habrá oportunidades. En el casode la educacióngerontológica, contar con personal cualificado quepueda ser multiplicador del conocimiento. El conocimiento objetivo de la vejez y de su proceso define la calidad de vida que tenga. Este bienestar vendrá del resultado de los déficits y/o los buenos hábitos en los que se hayan incurrido, durante todo el ciclo vital Aristizábal-Vallejo, (2007).

En este orden de ideas, la psicología de la tercera edad será sin lugar a dudas el reto en salud pública más importante de este siglo. Por lo tanto, a pesar delaaspiración universal de prolongar la vida, la sociedad desconoce muchos de los problemas de los adultos mayores, las cuestiones vinculadas al envejecimiento y las posibilidades que ofrece esta etapa de la vida, por la falta de información. De allí que, llegar a ser un área prioritaria en investigaciones científicas, generara: Líneas de investigación y aportes científicos para la atención de los adultos mayores.

La propuesta inicial de un modelo de intervención psicológica para adultos mayores, teniendo en cuenta el crecimiento de esta población, surgió de la necesidad de comprender e intervenir eficazmente sobre los mismos, y generar planes de acción que contribuyan al mejoramiento de su calidad de vida, a partir de una mirada integral a las necesidades propias de la población adulto mayor, su cultura, condiciones sociodemográficas y las investigaciones realizadas. De esta manera, se hace un llamado a las diferentes disciplinas que se interesan por el estudio del adulto mayor para que contribuyan al entendimiento de la complejidad de esta población y, sobre todo, al desarrollo de herramientas que permitan mejorar la vidadel adulto en todas sus áreas de funcionamiento (Uribe-Rodríguez \&Buela-Casal, 2003).

\section{Referencias bibliográficas}

Agencia Nacional de Evaluación de la Calidad y Acreditación (2005). Libro Blanco del Título del Grado en Psicología. Recuperado el 15 de diciembre de 2016, desde http://www.aneca.es/var/media/150356/ libroblanco_psicologia_def.pdf

Aristizábal, \& Vallejo, N. (2007). Estudio histórico comparado de la formación gerontológica en Psicología: Colombia y España. Tesis doctoral, Perspectiva histórica comparada y política de la educación. Programa de doctorado Facultad de Educación. Universidad de Salamanca. 
Carbajo, M. C. (2008).La Historia de la Vejez.Universidad de Valladolid, Ensayos, (18), 237-254.

Colegio Oficial de Psicólogos (2002). Psicología, psicólogos y envejecimiento: Contribución de la psicología y los psicólogos al estudio y la intervención sobre el envejecimiento. Revista Especializada en Gerontología, 37 268-275.

Comisión Nacional de Evaluación y Acreditación Universitaria del Ministerio de Educación, Ciencia y Tecnología de Argentina. (2005). Acreditación de la carrera de Especialización en Psicogerontología, de la Universidad Maimónides. Ley 24.521, la Resolución del Ministerio de Cultura y Educación N ${ }^{\circ} 1168 / 97$, la Ordenanza N034 - CONEAU y la Resolución $N^{\circ} 534$ - CONEAU - 01.

Conde, J. (1998). La gerontología y sus acciones e interacciones del equipo multiinterdisciplinario. En vv.aa. (Eds.): Gerontología 2000 (pp. 30-33). México: Praxis.

De la Fuente, R. (1999). El envejecimiento: una etapa del ciclo vital. Salud Mental, 22(5): 1-2.

EFPA (European Federation of Psychologists'Associations).(2008). Recomendaciones del Grupo de Trabajo Europeo en Psicogerontología. Infocop | 27/06/2008. http://www. infocop.es/view_article.asp?id=1929

Ericsson, E. H. ( 1963) Chilhoodandsociety. New York: Norton.

Fernández Ballesteros, R. (2007). Psicogerontología: su importancia como ámbito aplicado. Infocop 02/10/2007. http://www. infocop.es/view_article.asp?id=1539

Fernández Ballesteros, R. (2009). (Dir.) Psicogerontología: Perspectivas Europeas para un Mundo que Envejece. Madrid: Pirámide.
Fernández Lópiz, E. (2015). Sobre la Formación del Docente-Facilitador de Personas Mayores. Educação\&Realidade, 40 (1). Recuperado de: http://www.seer.ufrgs. br/index.php/educacaoerealidade/article/ view/45536/3221

Fernández, \& Ballesteros, R. (2001). Psicología y envejecimiento: retos internacionales en el siglo XXI: Intervención Psicosocial. Vol. $10, \mathrm{~N}^{\circ}{ }^{\circ}$ 3. Universidad Autónoma de Madrid

Fernández, \& Ballesteros, R. (Dir.) (2009b). Psicología de la vejez: una Psicogerontología aplicada. Madrid: Pirámide.

Fernández, R. (1992). Evaluación e intervención psicológica en la vejez. Barcelona: Ediciones Martínez Roca.

Fundación Chile. (2000). Proyecto Certificación de Competencias Laborales y calidad de la capacitación. Santiago de Chile: Presentación en PowerPoint. Consultado el 7 de mayo del 2002 Recuperado de: http://www.ilo.org/public/spanish/ region/ampro/cinterfor/temas/complab/ doc/otros/f_chile/sld001.htm

Galvanovsky, L. (1997) Psicología y envejecimiento. Editorial Mexico, S.A.

Gutiérrez, O. (2005). Educación y entrenamiento Basados en el Concepto de Competencia: Implicaciones para la Acreditación de los Programas de Psicología. Revista Mexicana de Psicología, 22. Número monográfico especial. Pp. 253-270. Con acceso el 02 de septiembre de 2005 en Recuperado de: http://www.psicologia. org.mx/articulo\%204.pdf

Hall, ST. (1922). Senescence, the last half of life. Nueva York: Appleton

Moragas, R. (2004) Gerontología social. Envejecimiento y calidad de vida. Barcelona: Editorial Herder. 
Moura, C.; Navarro, J.C; Wolf, L. \&Cabrol, M. (1998). La educación como catalizador del progreso: la contribución del Banco Interamericano de Desarrollo. Banco Interamericano de desarrollo, Departamento de Desarrollo sostenible. Con acceso el 30 de octubre de 2005 en Recuperado de: http:// www.iadb.org/sds/doc/edu-SantiagoS.pd

Organización Mundial de la Salud (2006). Género y envejecimiento. Recuperado de: http://www.paho.org/Spanish/AD/GE/genderageingsp.PDF. Accedido el 15 agosto 2006.

Orosa, F. T. (2003) Determinantes del desarrollo de la psicología de la vejez. Presupuestos. científicos en la Universidad del Adulto Mayor. La Habana: Editorial Félix Varela.

Reyes, L. (1996). Acercamientos tanatológicos al enfermo terminal y a su familia. México: Autor.

Universidad de Salamanca. España: Máster Universitario en Psicogerontología 2021/2022 [acceso 29/06/2021]. Disponible en: https://www.usal.es/master-psicogerontologia [ Links ]

Uribe-Rodríguez, A. F. \&Buela- Casal, G. (2003). Influencia del tipo de residencia sobre la depresión, el deterioro cognitivo y la calidad de vida en personas mayores. Granada: Universidad de Granada (Documento sin publicar).
Uribe-Rodríguez, A. F., Molina, J. M. \& González, L. (2006). Communication: Program of intervention on the quality of life, the depression and the cognitive deterioration in people who belong to groups of the third age. 26th International Congress of Applied Psychology.Atenas (Grecia).

WHO. Decade of Healthy Ageing. Ginebra: WHO; 2020 [acceso 27/08/2020]. Disponible en: Disponible en: https://www. who.int/initiatives/decade-of-healthy-ageing [ Links ]

Zarebski, G. (2005). Conferencia Inaugural. Primer Congreso Iberoamericano de Psicogerontología. Buenos Aires: Universidad Maimónides.

Zarebski, G. (2005). Hacia un Buen Envejecer. Buenos Aires: Univ. 32. Maimónides, Científica y Literaria.

Zarebski, G. (2015). Conferencia Inaugural. Sexto. Congreso Iberoamericano de Psicogerontología. La Paz, Bolivia: Universidad Maimónides. 